\title{
Preceding psychological factors and calorie intake in patients with type 2 diabetes: investigation by ecological momentary assessment
}

\author{
Shuji Inada ${ }^{1}$, Yoko lizuka ${ }^{2}$, Ken Ohashi ${ }^{3}$, Hiroe Kikuchi ${ }^{4}$, Yoshiharu Yamamoto ${ }^{5}$, Takashi Kadowaki ${ }^{6}$ and \\ Kazuhiro Yoshiuchi ${ }^{1^{*}}$ (D)
}

\begin{abstract}
Background: Psychological factors have been reported to have influence on the eating habits of patients with diabetes. However, previous studies have used questionnaires to investigate the association, and thus include recall bias. To overcome this disadvantage, ecological momentary assessment (EMA) can be used to record subjective symptoms and behavior in subjects' daily lives. Therefore, the aim of the present study was to investigate the influence of preceding psychological factors on calorie intake using computerized EMA for 6 months.

Methods: The participants were nine outpatients with type 2 diabetes, aged 34-72. They were instructed to use a personal digital assistant as an electronic diary for 6 months to record subjective symptoms, such as psychological stress, anxiety, and depressive mood, and the food and drink that they consumed. The association between a preceding psychological factor and calorie intake within 5 hours was investigated using multilevel modeling.

Results: Preceding psychological stress was positively associated with calorie intake from snacks. Preceding psychological stress, anxiety, and depressive mood were negatively associated with calorie intake from regular meals.

Conclusions: Preceding psychological factors influence the calorie intake of patients with type 2 diabetes. Understanding the role of these factors will be useful for developing psychological interventions to prevent overeating. Trial registration: The trial registration number: UMIN000002992. Date of registration: 2010/01/07.

Keywords: Ecological momentary assessment, Type 2 diabetes, Electronic food diary, Psychological factors, Multilevel modeling
\end{abstract}

\section{Background}

Psychological factors, such as anxiety and depressive mood, have been reported to have influence on the eating habits of patients with life-style diseases, such as diabetes, which raises the risks of cardiovascular diseases [1]. Many studies have used questionnaires to investigate the association between psychological factors and eating habits, as in this previous study. However, the use of questionnaires is problematic because of their potential

\footnotetext{
* Correspondence: kyoshiuc-tky@umin.ac.jp

${ }^{1}$ Department of Stress Sciences and Psychosomatic Medicine, Graduate School of Medicine, The University of Tokyo, 7-3-1 Hongo, Bunkyo-ku, Tokyo, Japan

Full list of author information is available at the end of the article
}

for recall bias [2]. To overcome this disadvantage, ecological momentary aqssessment (EMA), which avoids recall bias, has been used in the area of behavioral medicine to record subjective symptoms and behavior in subjects' daily lives.

Some previous studies have been done to investigate the association between psychosocial factors and food intake using EMA [3-6]; however, these studies included methodological problems. First, most [3-5] used paper-andpencil diaries to record subjective symptoms and food intake, while Tomiyama et al. [6] used an electronic diary (i.e. computerized EMA). Compared with EMA using paper-and-pencil diaries, computerized EMA has the advantage of avoiding 'faked compliance', i.e. disguised

(C) The Author(s). 2019 Open Access This article is distributed under the terms of the Creative Commons Attribution 4.0 International License (http://creativecommons.org/licenses/by/4.0/), which permits unrestricted use, distribution, and 
compliance by recording data at times other than those designated, by recording the time of data input [7]. In addition, the study by Tomiyama, which used computerized EMA, only assessed two days.

The aim of the present study was to do a long-term, quantitative investigation of the influence of preceding psychological factors on calorie intake, using computerized EMA for six months.

\section{Methods}

\section{Participants and procedures}

The participants were nine outpatients with type 2 diabetes diagnosed according to the current criteria of the Japan Diabetes Society [8] (three men; median age $=49$ years, range $=$ 34 to 72) who also took part in another study [9]. Inclusion criteria were age $\geq 20$ and an outpatient with type 2 diabetes at the University of Tokyo Hospital. Participants were excluded if they had HbA1c $\geq 8.4 \%$ (corresponding to $8.0 \%$ before converting to the NGSP value, which was the criterion for poorly controlled type 2 diabetes), had any active mental disorder, followed a restrictive diet with protein $\leq 0.5 \mathrm{~g} / \mathrm{kg} /$ day or salt $\leq 5 \mathrm{~g} /$ day due to another disease, had cognitive impairment, or had severe complications that interfered with self-care activities. After providing written informed consent, participants were instructed to record daily dietary intake using a PDA-based food diary, SELFOOD [10], and to record mood states, such as anxiety and depression, on a personal digital assistant (PDA) (WS020SH, Sharp Corp, Osaka, Japan, $135 \mathrm{~g}, 50 \times 116 \times 17.9 \mathrm{~mm}$, Windows Mobile 6.1) that was equipped with a 3.0 -in color liquid crystal display and a touch panel manipulated by a finger. They were asked to record as soon as possible after eating for 6 consecutive months. Anthropometric measurements, including height, body weight, and blood pressure, were performed at the beginning of the study. Clinical data (e.g. fasting plasma glucose level, hemoglobin Alc value, and duration of diabetes) were collected at their most recent visit with a health care provider. They visited their physician regularly, and continued to take the medications prescribed for their conditions during the study period.

\section{Dietary intake}

We developed an electronic food diary, "SELFOOD", for use in a personal digital assistant (PDA). Its accuracy has been confirmed by comparison with a 24$\mathrm{h}$ recall interview by registered dietitians [10]. SELFOOD includes a reference database with 423 color photographs of food and drink items and allows patients to add their own meals and recipes to the database if their nutrient information is given. SELFOOD calculates energy intake after every input. Calorie intake was summed on an input-by-input basis and converted to a daily bar graph with a horizontal line indicating the target value of daily calorie intake.

\section{Momentary psychological stress and mood states}

A PDA was used as electronic diary to record psychological stress, anxiety, and depressive mood [11]. The subjects were instructed on the use of the device and given manuals before the beginning of the study period. In addition, they practiced manipulating the device with the author until they became accustomed to its use.

The subjects used the PDAs for six consecutive months. Signal-contingent recordings were defined as recordings that were prompted with a beep as a signal [2], which was programmed to occur randomly within an interval of $30 \mathrm{mins}$, around 10:00 and 15:00. If the subjects did not enter a recording when the computer beeped, they were allowed to postpone input for $30 \mathrm{~min}$. Recordings not made within 30 min were cancelled. The subjects were also asked to record their psychological stress, anxiety, and depressive mood when they woke up and when they went to bed by choosing "waking up" or "going to bed" from the menu. After selecting a "going to bed" recording, to avoid sleep disturbance the computers suspended the signal-contingent recordings until a selected "wake up" recording was played. Signal-contingent recordings and recordings when waking up and going to bed were treated as scheduled recordings. Momentary psychological stress, anxiety, and depressive mood were rated with a visual analog scale (VAS) that ranged from 0 to 100, which was displayed on the screen.

\section{Statistical analysis}

Multilevel modeling was used for statistical analyses to investigate preceding psychological factors on dietary intake because the dataset in this study had a nested structure in which a number of recordings belonged to each participant. Pairs of preceding psychological factors and dietary intake were lagged in the range of -5 to $0 \mathrm{~h}$ [12]. The level of significance was set at 0.05 . SAS Proc Mixed (SAS 9.3, SAS Institute Inc., Cary, NC, USA) was used. Each dietary intake recording was paired with the preceding momentary recording of psychological stress and mood states. Dietary intake was treated as the dependent variable. Momentary psychological stress, anxiety, and depressive mood were separately modeled as the independent variable, either as a fixed or random effect. Preceding momentary recordings of psychological factors were recorded within five hours before dietary intake, and there was no dietary intake between a preceding momentary recording of psychological factors and the dependent variable of dietary intake. Using these stratified time lags, models with different combinations of independent variables (with/without a preceding psychological factor as a fixed/random effect) were tested. Goodness of fit was compared to determine the best-fit model with a-2 log likelihood function and $x^{2}$ test when one model was nested in the other; otherwise, Akaike's Information Criterion (AIC) was used. The level-1 (within-individual 
level) intercept was modeled as a random effect. In all analyses, the variance-covariance matrix ( $G$ matrix) was modeled as unstructured.

\section{Results}

\section{Subject characteristics}

The characteristics of subjects are shown in Table 1.

\section{Recording profiles}

In total, the data from 1353 days of recordings was available for analysis. The compliance rate for regular dietary intake was $77.2 \%$, while the compliance rate for signalcontingent recordings of psychological factors was $42.8 \%$. With regard to pairing dietary intake and preceding psychological factors, 1517 of 3667 dietary intakes could be paired with preceding psychological factors.

\section{Preceding psychological stress and dietary calorie intake}

Preceding psychological stress was significantly, negatively associated with dietary intake for lunch and dinner, but positively associated for snacks (Tables 2, 3 and 4). The final model is as follows:

Level 1 equation:

$$
\begin{aligned}
\mathrm{Y}_{\mathrm{ij}}= & \pi_{0 \mathrm{i}}+\pi_{1 \mathrm{i}} \text { Company }_{\mathrm{ij}}+\pi_{2 \mathrm{i}} \text { Place }_{\mathrm{ij}}+\pi_{3 \mathrm{i}} \text { Stress }_{\mathrm{ij}} \\
& +\varepsilon_{\mathrm{ij}}
\end{aligned}
$$

Level 2 equation:

$$
\begin{aligned}
& \pi_{0 \mathrm{i}}=\gamma_{00}+\zeta_{0 \mathrm{i}}\left(\pi_{0 \mathrm{i}}=\gamma_{00}++\gamma_{01} \text { TargetCal }_{\mathrm{i}}+\zeta_{0 \mathrm{i}}\right. \text { for din } \\
& \pi_{1 \mathrm{i}}=\gamma_{10} \\
& \pi_{2 \mathrm{i}}=\gamma_{20} \\
& \pi_{3 \mathrm{i}}=\gamma_{30}
\end{aligned}
$$

where $Y_{i j}$ is each momentary calorie intake for the ith patient; Company ${ }_{\mathrm{ij}}$ is a corresponding accompanying person, recorded as alone or with someone; Place $_{\mathrm{ij}}$ is the corresponding eating place; home, office/school, dining out, or other (e.g. outdoors); Stress $\mathrm{ij}_{\mathrm{ij}}$ is the corresponding preceding psychological stress; TargetCal ${ }_{i}$ is the target calorie intake for the ith patient. $\gamma_{00}$ is the average true value of mean calorie intake when all predictors are zero. $\pi_{1 \mathrm{i}}, \pi_{2 \mathrm{i}}$, and $\pi_{3 \mathrm{i}}$ are the individual i's slopes representing the effect of an

Table 1 Subject characteristics

\begin{tabular}{ll}
\hline Number (male/female) & $9(6 / 3)$ \\
\hline Age (years) & $49(34,72)$ \\
BMl $\left(\mathrm{kg} / \mathrm{m}^{2}\right)$ & $25.5(20.3,52.3)$ \\
Duration of illness (years) & $6(0.5,16)$ \\
Blood HbA1c (\%) & $7.4(6.2,8.2)$ \\
Medication (n) & 9 \\
Oral hypoglycemic agents & 4 \\
\hline Oral hypoglycemic agents + insulin & 4 \\
\hline
\end{tabular}

Values are given as median (minimum, maximum) unless otherwise stated accompanying person(s), eating place(s), or preceding psychological stress on momentary calorie intake, respectively, and $\gamma_{10}, \gamma_{20}$, and $\gamma_{30}$ are the average slopes. $\varepsilon_{\mathrm{ij}}$ and $\zeta_{0 \mathrm{i}}$ are residuals at each level. Including $\zeta_{0 \mathrm{i}}$ in the equation means that the intercept was modeled as random, which suggests that intercept can vary across individuals. The second level 2 equation included no residual, which means that the effect of an accompanying person(s), eating place(s), or preceding psychological stress on momentary calorie intake was modeled as a fixed effect.

\section{Preceding anxiety and dietary calorie intake}

Preceding anxiety was significantly, negatively associated with dietary intake only for breakfast (Table 5). The final model is as follows:

Level 1 equation:

$$
\begin{aligned}
\mathrm{Y}_{\mathrm{ij}}= & \pi_{0 \mathrm{i}}+\pi_{1 \mathrm{i}} \text { Company }_{\mathrm{ij}}+\pi_{2 \mathrm{i}} \text { Place }_{\mathrm{ij}}+\pi_{3 \mathrm{i}} \text { Anxiety }_{\mathrm{ij}} \\
& +\varepsilon_{\mathrm{ij}}
\end{aligned}
$$

Level 2 equation:

$$
\begin{aligned}
& \pi_{0 \mathrm{i}}=\gamma_{00}+\zeta_{0 \mathrm{i}} \\
& \pi_{1 \mathrm{i}}=\gamma_{10} \\
& \pi_{2 \mathrm{i}}=\gamma_{20} \\
& \pi_{3 \mathrm{i}}=\gamma_{30}
\end{aligned}
$$

where $Y_{i j}$ is each momentary calorie intake for the ith patient; Company $\mathrm{ij}_{\mathrm{ij}}$ is a corresponding accompanying persson, either alone or with someone; Place $_{i j}$ is the corresponding eating place, home, office/school, dining out, or other; Anxiety ${ }_{\mathrm{ij}}$ is the corresponding preceding anxiety.

\section{Preceding depressive mood and dietary calorie intake}

Preceding depressive mood was significantly, negatively associated with dietary intake only for lunch (Table 6). The final model is as follows:

Level 1 equation:

$$
\begin{aligned}
\mathrm{Y}_{\mathrm{ij}}= & \pi_{0 \mathrm{i}}+\pi_{1 \mathrm{i}} \text { Company }_{\mathrm{ij}}+\pi_{2 \mathrm{i}} \text { Place }_{\mathrm{ij}} \\
& +\pi_{3 \mathrm{i}} \text { Depression }_{\mathrm{ij}}+\varepsilon_{\mathrm{ij}}
\end{aligned}
$$

Level 2 equation:

$$
\begin{aligned}
& \pi_{0 \mathrm{i}}=\gamma_{00}+\zeta_{0 \mathrm{i}} \\
& \pi_{1 \mathrm{i}}=\gamma_{10} \\
& \pi_{2 \mathrm{i}}=\gamma_{20} \\
& \pi_{3 \mathrm{i}}=\gamma_{30}
\end{aligned}
$$

where $Y_{i j}$ is each momentary calorie intake for the ith patient; Company $\mathrm{ij}_{\mathrm{ij}}$ is a corresponding accompanying person, either alone or with someone; Place $_{i j}$ is the corresponding eating place, home, office/school, dining out, or other; Depression ${ }_{i j}$ is the corresponding preceding depressive mood. 
Table 2 Effect of preceding psychological stress on calorie intake, for lunch

\begin{tabular}{lll}
\hline Lunch & Coefficient (S.E.) & $F$ value \\
\hline Intercept $(\gamma 00)$ & $565.71(47.45)$ & \\
Accompanying person $(\gamma 10)$ & & $F(1,7)=19.04$ \\
$\quad$ Alone & $446.84(35.59)$ & \\
$\quad$ Someone & $525.91(36.04)$ & \\
Place $(\gamma 20)$ & & $F(3,10)=11.39$ \\
$\quad$ Home & $489.18(34.57)^{a}$ & \\
Office/school & $410.24(41.90)^{a}$ & \\
Dining out & $565.57(35.39)$ & \\
Other & $480.52(45.09)$ & \\
Stress $(\gamma 30)$ & $-1.7259(0.4369)$ & $F(1,467)=15.61$ \\
\hline
\end{tabular}

aSignificant difference with "Dining out" ( $p<0.05$ for all using Tukey-Kramer's correction)

\section{Discussion}

Using computerized EMA for 6 months, significant within-individual relationships between preceding psychological factors and calorie intake within 5 hours were shown in patients with type 2 diabetes. Although a causal relation could not be established, our results support the possibility that psychological factors can influence the calorie intake of patients with type 2 diabetes, which has not been investigated previously on a daily-life basis.

In the present study, the results showed differences in the association between preceding psychological factors and calorie intake among types of food. With regard to snacks, we showed that preceding psychological stress was positively associated with calorie intake. This result is consistent with the results of previous studies $[3,5,6]$. In the study by Lowe et al. [3], calorie intake from

Table 3 Effect of preceding psychological stress on calorie intake, for dinner

\begin{tabular}{|c|c|c|c|}
\hline Dinner & Coefficient (S.E.) & Fvalue & $P$ value \\
\hline Intercept (ү00) & $-256.15(297.61)$ & & 0.4179 \\
\hline Accompanying person $(\gamma 10)$ & & $F(1,6)=5.58$ & 0.0562 \\
\hline Alone & 484.64(40.44) & & \\
\hline Someone & $548.53(37.51)$ & & \\
\hline Place $(\gamma 20)$ & & $F(3,4)=12.26$ & 0.0174 \\
\hline Home & $577.45(30.41)$ & & \\
\hline Office/school & $366.44(57.04)^{* \#}$ & & \\
\hline Dining out & $684.19(44.51)$ & & \\
\hline Other & $438.27(60.66)^{*}$ & & \\
\hline Stress $(\gamma 30)$ & $-1.2209(0.5118)$ & $F(1,385)=12.26$ & 0.0175 \\
\hline Target calorie (ү01) & $0.5106(0.1881)$ & $F(1,7)=7.37$ & 0.0300 \\
\hline
\end{tabular}

snacks increased when subjects with obesity were in a negative mood, although the study did not investigate the relation proportionally between negative mood and calorie intake. O'Connor et al. [5] reported a positive association between daily hassles and calorie intake from snacks, using only end-of-day assessments that did not include momentary psychological factors. Tomiyama et al. [6] used computerized EMA in a short two-day report that found preceding negative mood to be positively associated with calorie intake of snacks. Because the results of studies using such different methods were consistent, psychological stress would seem to increase calorie intake of snacks. Eating snacks may be a coping mechanism for reducing psychological stress [13].

With regard to regular meals, preceding psychological stress, anxiety, and depressive mood were negatively associated with calorie intake. Negative mood may cause

Table 4 Effect of preceding psychological stress on calorie intake, for snacks

\begin{tabular}{|c|c|c|c|}
\hline Snacks & Coefficient (S.E.) & $F$ value & $P$ value \\
\hline Intercept (ү00) & $93.54(48.68)$ & & 0.0961 \\
\hline Accompanying person $(\gamma 10)$ & & $F(1,5)=7.36$ & 0.0422 \\
\hline Alone & 100.34(41.84) & & \\
\hline Someone & 154.91(41.99) & & \\
\hline Place $(\gamma 20)$ & & $F(3,7)=6.62$ & 0.0188 \\
\hline Home & 158.14(41.11) & & \\
\hline Office/school & $69.24(43.62)^{* \#}$ & & \\
\hline Dining out & 189.18(49.57) & & \\
\hline Other & $93.93(44.07)^{\#}$ & & \\
\hline Stress ( $ү 30)$ & $1.2513(0.5403)$ & $F(1,186)=5.36$ & 0.0216 \\
\hline
\end{tabular}


Table 5 Effect of preceding anxiety on calorie intake, for

\begin{tabular}{|c|c|c|c|}
\hline Breakfast & Coefficient (S.E.) & $F$ value & $P$ value \\
\hline Intercept (ү00) & $596.20(50.58)$ & & $<0.0001$ \\
\hline Accompanying person $(\gamma 10)$ & & $F(1,4)=7.68$ & 0.0502 \\
\hline Alone & $418.98(35.84)$ & & \\
\hline Someone & $489.73(37.17)$ & & \\
\hline Place (ү20) & & $F(3,5)=4.45$ & 0.0708 \\
\hline Home & $413.79(29.72)$ & & \\
\hline Office/school & $375.90(66.19)$ & & \\
\hline Dining out & $516.49(49.69)$ & & \\
\hline Other & $511.26(51.49)$ & & \\
\hline Anxiety (ү30) & $-1.8047(0.7633)$ & $F(1,395)=5.59$ & 0.0185 \\
\hline
\end{tabular}

not only emotional eating, but appetite loss mediated by autonomic reaction. Because the patients were following diet therapy advice, the calorie intake in their regular meals might have been affected by a physical reaction rather than by an emotional reaction. The results of previous studies were not consistent regarding the association between psychological factors and calorie intake. Lowe et al. [3] found no association between negative mood and the calorie intake from regular meals, while Patel et al. [4] reported that the calorie intake from regular meals was greater for "negative mood" than for "neutral mood". Because all of the subjects were women in the two previous studies, it would be difficult to compare them to the results of the present study. Therefore, future studies will necessary to confirm the association between preceding psychological factors and regular meals.

When controlling for psychological factors, calorie intake was generally greater when eating with than without another person(s) and when eating out than when eating at home. This result supports the results shown in a previous study that calorie intake increased in social situations [4].

Table 6 Effect of preceding depressive mood on calorie intake, for lunch

\begin{tabular}{|c|c|c|c|}
\hline Lunch & Coefficient (S.E.) & $F$ value & $P$ value \\
\hline Intercept (ү00) & $564.61(43.27)$ & & $<0.0001$ \\
\hline Accompanying person $(\gamma 10)$ & & $F(1,7)=22.26$ & 0.0022 \\
\hline Alone & $442.80(29.85)$ & & \\
\hline Someone & $528.15(30.60)$ & & \\
\hline Place (ү20) & & $F(3,10)=11.70$ & 0.0013 \\
\hline Home & $491.43(28.81)^{*}$ & & \\
\hline Office/school & $414.13(37.15)^{*}$ & & \\
\hline Dining out & $569.16(29.91)$ & & \\
\hline Other & $467.18(40.71)$ & & \\
\hline Depressive mood (ү30) & $-3.6393(0.9826)$ & $F(1,467)=13.72$ & 0.0002 \\
\hline
\end{tabular}

"Significant difference with "Dining out" ( $p<0.05$ for all using Tukey-Kramer's correction)
There were some limitations in the present study. First, the sample size was small. Second, most of the patients in the present study were men. Therefore, future studies with more patients including more men and women will be necessary. Also, there was no control group of healthy people, which makes it impossible to expand the results in the present study to healthy people. In addition, some of the dietary intake could be paired with preceding psychological factors, which might be subject to selection bias.

\section{Conclusions}

Preceding psychological factors influence the calorie intake of patients with type 2 diabetes. Better understanding these factors will be helpful for developing psychological interventions to prevent overeating.

\section{Abbreviations}

AIC: Akaike's information criterion; EMA: ecological momentary assessment; $\mathrm{HbA1c}$ : hemoglobin A1c; PDA: portable digital assistant; VAS: visual analog scale

\section{Acknowledgements}

The authors thank all of the study participants. Gratitude is also expressed to Dr. Kojima from Sharp Corporation for his technical help in developing the software in Widows Mobile.

\section{Authors' contributions}

SI designed the study, analyzed the data, performed the statistical analysis, interpreted the results, and drafted the manuscript. $\mathrm{YI}$ and $\mathrm{KO}$ helped design the study and collect data. HK, YY and TK helped design the study. KY conceived of the present idea and helped design the study, analyze the data, interpret the results, and draft the manuscript. All authors have read, modified and approved the final manuscript.

\section{Funding}

The University of Tokyo (person in charge: Dr. Yoshiuchi) conducted cooperative research with Sharp Cooperation.

\section{Availability of data and materials}

We are not able to share our data because sharing data is not permitted by our hospital or the ethics committee.

\section{Ethics approval and consent to participate}

All procedures performed in studies involving human participants were in accordance with the ethical standards of the institutional and/or national research committee and with the 1964 Helsinki declaration and its later amendments or comparable ethical standards. The study protocol was approved by the ethics committee of the Graduate School of Medicine, The University of Tokyo (ID: 2728). Informed consent was provided by all individual participants included in the study.

\section{Consent for publication}

Not applicable.

\section{Competing interests}

Author Yoshiuchi K. received research grants from Sharp Cooperation for the submitted work

\section{Author details}

${ }^{1}$ Department of Stress Sciences and Psychosomatic Medicine, Graduate School of Medicine, The University of Tokyo, 7-3-1 Hongo, Bunkyo-ku, Tokyo, Japan. ${ }^{2}$ Department of Diabetes and Metabolic Diseases, Graduate School of Medicine, The University of Tokyo, 7-3-1 Hongo, Bunkyo-ku, Tokyo, Japan. ${ }^{3}$ Department of Internal Medicine, National Cancer Center Hospital, 5-1-1 Tsukiji, Chuo-ku, Japan. ${ }^{4}$ Department of Psychosomatic Medicine, Center 
Hospital, National Center for Global Health and Medicine, 1-21-1 Toyama, Shinjuku-ku, Tokyo, Japan. ${ }^{5}$ Educational Physiology Laboratory, Graduate School of Education, The University of Tokyo, 7-3-1 Hongo, Bunkyo-ku, Tokyo, Japan. ${ }^{6}$ Department of Diabetes and Metabolic Diseases and Department of Prevention of diabetes and lifestyle-related diseases, Graduate School of Medicine, The University of Tokyo, 7-3-1 Hongo, Bunkyo-ku, Tokyo, Japan.

Received: 31 May 2019 Accepted: 20 August 2019

Published online: 04 September 2019

\section{References}

1. Bonnet F, Irving K, Terra J, Nony P, Berthezène F, Moulin P. Anxiety and depression are associated with unhealthy lifestyle in patients at risk of cardiovascular disease. Atherosclerosis. 2005;178:339-44.

2. Stone AA, Shiffman SS. Ecological momentary assessment (EMA) in behavioral medicine. Ann Behav Med. 1994;16:199-202.

3. Lowe MR, Fisher EB. Emotional reactivity, emotional eating, and obesity: a naturalistic study. J Behav Med. 1983;6:135-49.

4. Patel KA, Schlundt DG. Impact of moods and social context on eating behavior. Appetite. 2001;36:111-8.

5. O'Connor DB, Jones F, Conner M, McMillan B, Ferguson E. Effects of daily hassles and eating style on eating behavior. Health Psychol. 2008;27:S20-31.

6. Tomiyama AJ, Mann T, Comer L. Triggers of eating in everyday life. Appatite. 2009;52:72-82.

7. Stone AA, Shiffman S, Schwaltz JE, Broderick JE, Hufford MR. Patient noncompliance with paper diaries. BMJ. 2002;324:1192-3.

8. Seino Y, Nanjo K, Tajima N, Kadowaki T, Kashiwagi A, Araki E, et al. Report of the committee on the classification and diagnostic criteria of diabetes mellitus: the Committee of the Japan Diabetes Society on the diagnostic criteria of diabetes mellitus. Diabetol Int. 2010;1:2-20.

9. Inada S, Yoshiuchi K, lizuka Y, Ohashi K, Kikuchi H, Yamamoto Y, et al. Pilot study for the development of a self-care system for type 2 diabetes patients using a personal digital assistant (PDA). Int J Behav Med. 2016;23:295-9.

10. Fukuo W, Yoshiuchi K, Ohashi K, Togashi H, Sekine R, Kikuchi H, et al. Development of a hand-held personal digital assistant-based food diary with food photographs for Japanese subjects. J Am Diet Assoc. 2009:109:1232-6.

11. Kikuchi H, Yoshiuchi K, Miyasaka N, Ohashi K, Yamamoto Y, Kumano H, et al. Reliability of recalled self-report on headache intensity: investigation using ecological momentary assessment technique. Cephalalgia. 2006;26:1335-43.

12. Kikuchi $\mathrm{H}$, Yoshiuchi $\mathrm{K}$, Ando $\mathrm{T}$, Yamamoto $\mathrm{Y}$. Influence of psychological factors on acute exacerbation of tension-type headache: investigation by ecological momentary assessment. J Psychosom Res. 2015;79:239-42.

13. Macht M. How emotions affect eating: a five-way model. Appetite. 2008:50:1-11.

\section{Publisher's Note}

Springer Nature remains neutral with regard to jurisdictional claims in published maps and institutional affiliations.

Ready to submit your research? Choose BMC and benefit from:

- fast, convenient online submission

- thorough peer review by experienced researchers in your field

- rapid publication on acceptance

- support for research data, including large and complex data types

- gold Open Access which fosters wider collaboration and increased citations

- maximum visibility for your research: over $100 \mathrm{M}$ website views per year

At $\mathrm{BMC}$, research is always in progress.

Learn more biomedcentral.com/submissions 